\title{
THINKING DIFFERENTLY: ZIG-BEE BASED SMART HOME AUTOMATION
}

\author{
Hemant Pant ${ }^{1}$, Jyoti Thakur ${ }^{2}$, Anil Kumar ${ }^{3}$, H.P.S Kang ${ }^{4}$ \\ ${ }^{1}$ M-Tech, Instrumentation, Punjab University, Chandigarh, India \\ ${ }^{2} M-T e c h$, Instrumentation, Punjab University, Chandigarh, India \\ ${ }^{3}$ Assistant Professor, UCIM, Punjab University, Chandigarh, India \\ ${ }^{4}$ Assistant Professor, UCIM, Punjab University, Chandigarh, India
}

\begin{abstract}
The technology for Home Automation is present for many decades, but still very few people have actually automated their homes. It was thought that the biggest barriers are their cost and incompatibility with other manufacturers. To solve both of these problems Zig-bee protocol was established. Zig-bee chips are cheap and with the formation of the Zig-bee alliance incompatibility is not an issue. But still the Home Automation (HA) is not as common as what was expected. This paper addresses the issues which must be considered to make this technology as common as cell-phones. Based on the opinions given in this paper hardware has also been implemented.
\end{abstract}

Keywords: Home-Automation, Zig-bee, 802.15.4, IR-motion sensor, LM-35 temperature sensor.

\section{INTRODUCTION}

There are many wireless technologies available for Home automation like Z-Wave, Zig-bee, INSTEON, IP-based technology, Wavenis each having their own advantages and disadvantages.[7] but Zig-bee has its own set of advantages and undoubtedly has became the first choice for Home Automation.

Zig-bee is a protocol having all the features of 802.15.4 plus some other features. It is one of the fewest technologies which support mesh topology. Its other feature includes low cost, interoperability, low power consumption, reliability and it supports large number of nodes $(65000+$ theoretically). The data is transmitted in $2.4 \mathrm{Ghz}$ band with 16 available channels which reduces the chances of interference with other Zig-bee nodes. It also has a strong IEEE 802.15.4 protocol which allows it to send acknowledgements and retries thus making the process of transmission more reliable. To overcome the problem of incompatibility Zigbee alliance was founded in 2002 and it has more than 200 big companies. These companies provide Zig-bee based products all of which are compatible with each other.[1]

\subsection{Literature Survey}

1) After studying the survey done by A.J. Bernheim Brush and his team [2], they have addressed the four reasons that why the Home Automation has not been widely accepted.

2) Again the problem of implementation of HA was discussed by Armando Roy Delgado, Rich Picking and Vic Grout [3] the reasons being incompatibility with other manufacturers, scalability etc.
The work done by Michal VARCHOLA, Miloš DRUTAROVSKÝ clearly shows that indeed the Zig-bee's are interoperable.[4] And other issues have also been solved by the protocol called Zig-bee. Many papers has also been written on Zig-bee and work has also been implemented like that by Mitali and her mates[5] by creating a Zig-bee based voice control system or the work done on green house using Zig-bee by Y.R.Dhumal and J.S.Chitode[6]. But here we want to ask that it's been almost 12 years since the formation of Zig-bee alliance in 2002 and so many designs has been discussed as well as implemented, but still the Home Automation has not been widely accepted. Maybe this paper may provide the answer.

\subsection{Nodes of Zig-bee.}

Zig-bee nodes can function either as a coordinator, router or as an end device. Their functions are as follows:-

1) Coordinator:-This Zig-bee node is responsible to set a network. If this goes down the complete system goes down.(not in mesh topology)

2) Router:- If the data cannot pass between two Zig-bee nodes directly due to their range limitations. They must pass the data to the nearby Zig-bee node known as router. These Zig-bee nodes generally do not have any sensors directly connected to it and are responsible only for routing of data.

3) End Device:-All the Zig-bee node which has the sensors connected to it and do not route the data can be considered as the End device. 


\section{Network Topologies}

There are many ways in which the Zig-bee nodes many be connected. Each have thei own advantages and disadvantages. These schemes includes:-

1) Bus

2) Ring

3) Star

4) Tree

5) Mesh

Out of these five topologies Mesh is the most reliable as there is no fixed coordinator and even if one node does not work the data can be sent through any other node. As already stated Zig-bee can support mesh topology. IEEE other protocols including Bluetooth and Wi-Fi does not support mesh topology. How the nodes in the mesh topology is connected has shown in the figure 1 .

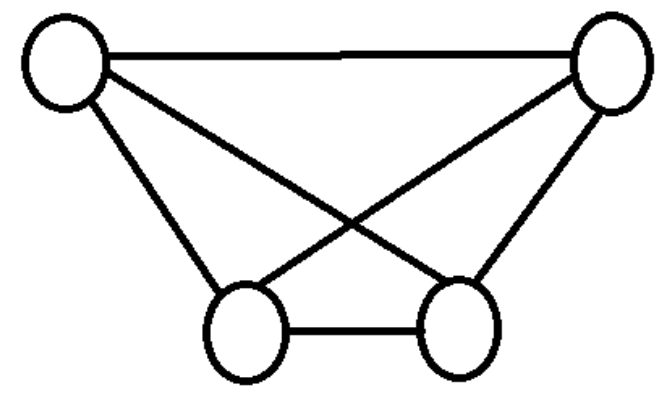

Fig:-1 Mesh Topology

\subsection{Zig-Bee Products}

There are many Zig-bee based products. The manufacturers of these products include LG electronics, Honeywell, Digiinternational, Samsung Electronics, Texas Instrument.[3]. The products can be seen in Figure 2 all of these products has one Zig-bee module per device. But here the question arises why one Zig-bee chip per product?

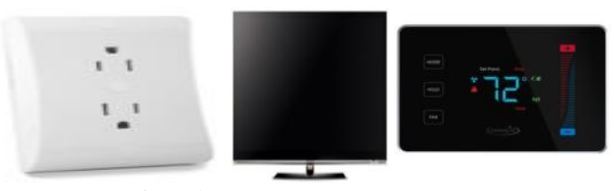

Fig-:2 Zig-Bee Products.

\subsection{Thinking Differently}

Although Zigbee is a cheap wireless technology with many other advantages but putting one Zig-bee chipset in each of the device may not be "The most Brilliant Idea" because by doing so, the cost of the Home Automations as a whole again comes up. It was earlier thought that one may automate his home by purchasing one or two products at a time and increase the automation with time. But in our opinion one room can be automated at a time depending on the loads connected in that room. The idea is that the ZigBee based wall sockets can be manufactured with relay outputs and many devices can be controlled through one X-
Bee module. Thereby decreasing the cost of the complete Home Automation considerably. Although many hobbyist have implemented this idea but still this scheme is not implemented in Home Automation.

So we have developed the hardware to control at least 2devices and also share the other data analog as well as digital (to show that any sensor can be used) through the sensors using only two X-BEE module.

\section{THE HARDWARE}

The hardware part of the work involves circuit design's that can pass the information back and forth from $\mathrm{x}$-bee on remote control to the $\mathrm{x}$-bee mounted on the relay module, and thus the action can be taken accordingly. The circuitry design involve microcontroller's, x-bee's, voltage-regulator, motion sensor, temperature sensor, lead wire, switches, resistors and PCB on which circuit is built.

By pressing the switch on the remote control appropriate action will be taken for example:

1) Temperature can be checked remotely and certain action can be initiated.

2) Status of various appliances can be checked (whether on or off).

3) Appliance can be switched ON or OFF.

4) Motion's detected by motion sensor will be informed remotely.

The ATmega-328 has been used to complete the hardware. It has been programmed using the Arduino platform which has pre-written libraries to work with most of the hardware parts. The X-bee's were programmed using X-CTU, a software provided by Digi International. The baud rate of both the microcontroller as well as well as X-bee has been set to $9600 \mathrm{bits} / \mathrm{sec}$. Figure-3 shows the remote control and the module to be controlled. Here led's are acting as the relay switches. LM-35 temperature sensor and PIR motion detector can also be seen in the provided figure. The responses of the hardware can be seen in Figure 4. Here two sensors PIR motion sensor as well as LM-35 temperature sensor has been used. Motion sensor gives digital output and analog sensor gives analog output. Using these two sensors it is obvious that most of the other sensors can also be used in this system as their output is either analog or digital.

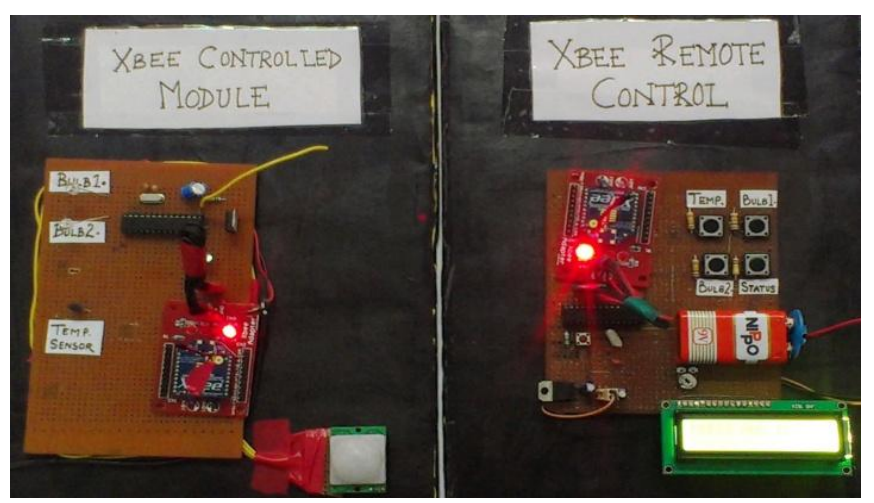

Fig -3: Hardware Setup 


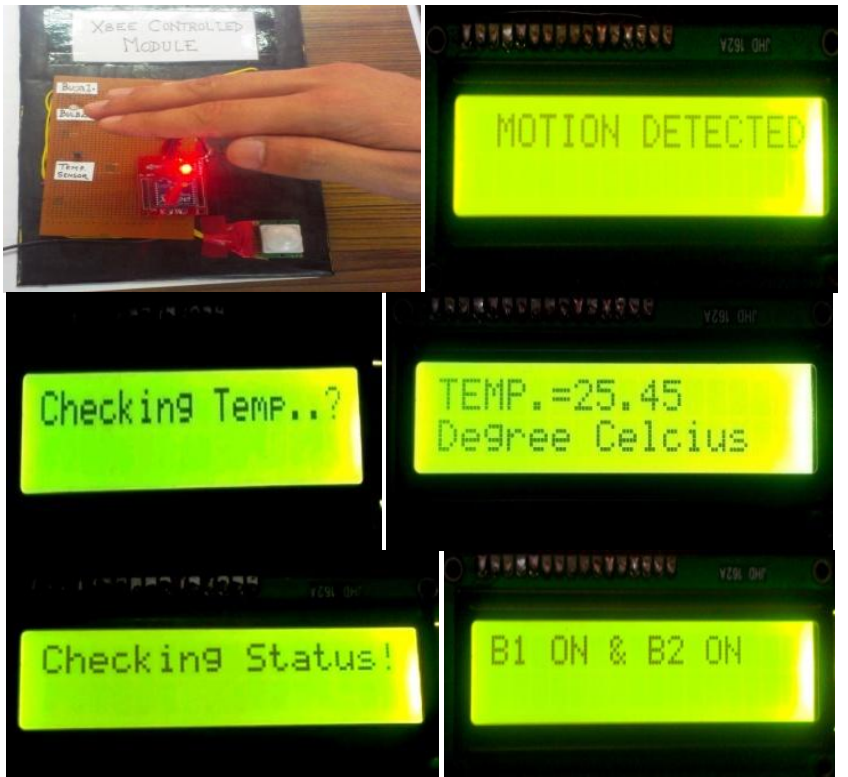

Fig-4 Responses Seen on LCD on Remote Control.

\section{CONCLUSIONS}

Zigbee is one of the cheapest and most reliable technology so far that has been developed as required for the applications of home automation. But using the X-Bee modules the right way is also our responsibility. Therefore rather than using one X-Bee module per device we should concentrate on the methods where we can use one X-Bee module for more than one device.

\section{ACKNOWLEDGEMENTS}

We are thankful to Punjab University, UCIM department for providing us the lab and necessary equipments for our experiments and our guides Assistant Professor Mr. H.P.S Kang and Assistant Professor Mr. Anil Kumar for their support and guidance.

\section{REFERENCES}

[1]. Basma M. Mohammad El-Basioni, Sherine M. Abd Elkader and Mahmoud AbdelmonimFakhreldin "Smart Home Design using Wireless Sensor Network and Biometric Technologies" International Journal of Application or Innovation in Engineering \& Management (IJAIEM) Volume 2, Issue 3, March 2013

[2]. Home Automation in the Wild: Challenges and Opportunities A.J. Bernheim Brush, Bongshin Lee, Ratul Mahajan, Sharad Agarwal, Stefan Saroiu, Colin Dixon* Microsoft Research, *University of Washington \{ajbrush, bongshin, ratul, sagarwal, ssaroiu\}@microsoft.com, ckd@cs.washington.edu

[3]. Remote-Controlled Home Automation Systems with Different Network Technologies Armando Roy Delgado, Rich Picking and Vic Grout Centre for Applied Internet Research (CAIR), University of Wales, NEWI, Wrexham, UK.

[4]. ZIGBEE BASED HOME AUTOMATION WIRELESS SENSOR NETWORK Michal VARCHOLA, Miloš DRUTAROVSKÝ Department of Electronics and

Multimedia Communications, Technical University of Košice, Park Komenského 13, 04120 Košice, Slovak Republic.

[5]. The Design and Implementation of Voice Controlled Wireless Intelligent Home Automation System Based on ZigBee Mitali Patil ,Ashwini Bedare Varsha Pacharne Computer Engineering, Computer Engineering Computer Engineering University of Pune, India University of Pune, India University of Pune, India.

[6]. Green House Automation using Zigbee and Smart Phone Y.R.Dhumal, J.S.Chitode Electronics Department \& Bharati Vidyapeeth University. Electronics Department \& Bharati Vidyapeeth University . India. India.

[7]. Wireless Home Automation Networks: A Survey of Architectures and Technologies by Carles Gomez and Josep Paradells, Technical University of Catalonia.

\section{BIOGRAPHIES}

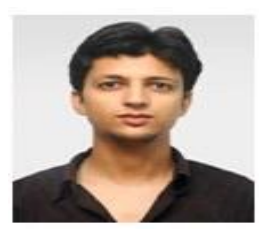

systems.

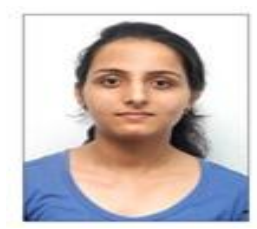

transmission lines.
Mr. Hemant Pant is B-Tech in Electrical Engineering and M-Tech in Instrumentation from Punjab University, Chandigarh. The author has keen interests in microcontrollers, PLC, wireless technology and embedded

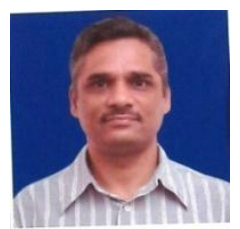

Mr. Anil Kumar is an Assistant Professor in UCIM Department Punjab University, Chandigarh, India. His research interests include Signal processing, transducers and nano -particles.

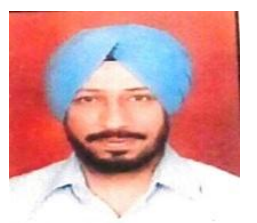

Mr. H.P.S Kang is an Assistant Professor in UCIM Department, Punjab University Chandigarh, India. His research interests include microelectronics and analytical instruments. 